\title{
Birth to Discharge: Neonatal Simulation Clinical Experiences
}

\author{
Cheryl L. DeGraw*
}

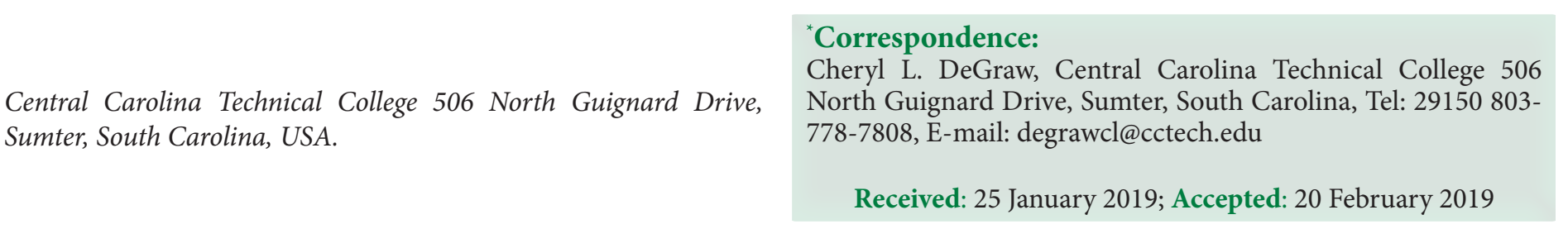

Citation: Cheryl L. DeGraw. Birth to Discharge: Neonatal Simulation Clinical Experiences. J Pediatr Neonatal. 2019; 1(1): 1-5.

ABSTRACT
With an increase in nursing programs to combat the projected nursing shortage, there is more competition for
clinical sites for Obstetric-Neonatal clinical rotations. Obstetric and neonatal simulation clinical experiences are
being substituted for hospital clinical rotations and for lack of patients during clinical rotations. A technical college
in Southeastern United States is using five-hour simulation clinical experiences, in which Obstetric and Neonatal
high-fidelity manikins are utilized, as substitutes for hospital clinical rotations or lack of Obstetric-Neonatal patients.
Neonatal nursing care is related to the type of disorder the pregnant patient is admitted with to the Antepartum/
Intrapartum Simulation Laboratory Hospital Unit. Three patient scenarios are used for the clinical experiences:
neonates born to mothers with gestational diabetes, preeclampsia, or preterm labor. Neonatal complications such
as hypoglycemia and prematurity are incorporated into the scenarios. Nursing students use fetal monitoring to
identify fetal complications to provide appropriate nursing care to the neonate after delivery. This use of simulation
clinical experiences has been endorsed by the National Council of State Boards of Nursing and the National League
of Nursing as a substitute for hospital clinical rotations. The significance of using Obstetric-Neonatal simulation
clinical experiences is increased understanding by nursing students of the entire antepartum through postpartum
nursing care and discharge process. Using the high-fidelity manikins and fetal monitoring, nursing students can
obtain hands-on experience when unable to obtain the clinical experience due to lack of a hospital rotation site or
lack of patients during their hospital clinical rotation.

\section{Keywords}

Neonatal, High-fidelity manikins, Simulation clinical experiences.

\section{Introduction}

Employment of registered nurses is projected to grow 15 percent from 2016 to 2026, much faster than the average for all occupations [1] (AACN, 2018). Growth will occur for several reasons, including an increased emphasis on preventive care; growing rates of chronic conditions, such as diabetes and obesity; and demand for healthcare services from the baby-boom population, as they live longer and more active lives [2] (Bureau of Labor Statistics, 2018). Due to the projected growth in employment, nursing programs have increased throughout the United States.

With the increase in nursing programs, there is a shortage in clinical sites for nursing students to learn Obstetric and Neonatal nursing. In small rural hospitals there may not be enough patients for nursing students to care for. Nursing programs in colleges and universities are using simulation laboratories to teach Obstetric and Neonatal nursing to improve nursing student's understanding of antepartum through postpartum nursing care. The use of simulation clinical experiences (SCEs) has been endorsed by the National Council of State Boards of Nursing and the National League of Nursing as a substitute for hospital clinical rotations [3] (Alexander, 2018, Spector, 2018). Simulation laboratories are equipped to represent hospital settings of Labor and Delivery, Postpartum, and Newborn Nursery units. Obstetric and Neonatal high-fidelity manikins are used for the patients.

\section{Method}

The SCEs used at the participating college are five-hour clinical experiences, including pre-briefing; antepartum, intrapartum, postpartum, and neonatal nursing care; and debriefing after completion of the simulation. Neonatal nursing care is related to the type of disorder the pregnant patient is admitted to the Simulation Laboratory Hospital Unit with. 
One of three patient scenarios is used for the SCEs: neonates born to mothers with gestational diabetes, preeclampsia, or preterm labor. Fetal monitoring is utilized to identify any fetal complications. Neonatal complications such as hypoglycemia and prematurity are incorporated into the SCEs.

\section{Student Preparation}

To prepare nursing students for the scenarios, a two-hour skill review is completed to include initiating intravenous access, performing venipunctures to draw blood for laboratory studies, administering medications, inserting indwelling urinary catheters, calculating intake and output, applying dressings, and inserting nasogastric tubes. A two-hour class is taught on fetal monitoring with emphasis on reading fetal monitor strips. In addition to these reviews, five-hours are spent in the simulation laboratory learning to use the electronic medication cart; intravenous pump; fetal monitoring; and assessing the antepartum, intrapartum, postpartum, and neonatal manikins. Prior to the SCEs, nursing students are given objectives to accomplish during the experience, see Appendix A.

\section{Simulated Clinical Experience}

During the half hour pre-briefing, nursing students choose from one of three notebooks containing the patient's medical records of an antepartum patient with gestational diabetes, preterm labor, or preeclampsia. The SCEs is the patient nursing students choose. Nursing students discuss how they will work as a team to complete the provider's orders.

The antepartum and intrapartum SCEs runs for one hour, 15 minutes from hospital admission through delivery of the fetus. During the SCEs, nursing students assess the obstetric patient and the fetus, obtain blood for laboratory studies and analyze the results, administer intravenous fluids and appropriate medications, perform skills as indicated, and assist with the fetal delivery. After delivery, a short debriefing is discussed while the Simulation Coordinator resets the manikin.

The postpartum SCEs runs for one hour, 15 minutes through the postpartum stay and prepares the patient and neonate for discharge. During the SCEs, the neonate has respiratory distress and hypoglycemia. Nursing students are required to perform appropriate nursing care based on their neonatal assessment. The obstetric patient will have a postpartum hemorrhage and may have a seizure. Again, nursing students are required to perform appropriate nursing care. Nursing faculty complete performance evaluations during the SCEs and share their observations with nursing students during the debriefing, see Appendix B and
Appendix C.

After the postpartum SCEs, one hour is spent in debriefing. Nursing students discuss how they performed during the SCEs. They observe the recording of the SCEs to evaluate the nursing care they performed. At the end of the debriefing, an evaluation form is completed by the nursing students, see Appendix D.

\section{Results}

For this study, validity is measured to determine if the SCEs perform as they are intended to, which is to improve student's understanding of the entire antepartum through postpartum nursing care and discharge process. The SCEs are as realistic as possible with the use of high fidelity manikins and provider's orders which are evidence-based from hospitals used for clinical rotations. Nursing students can perform evidence-based nursing care in a controlled situation.

Reliability is not measured as the SCEs is not a graded experience. Three faculties are involved in evaluating the SCEs; therefore, evaluations may differ based on their personal biases when evaluating nursing students.

Evaluations from nursing students were positive. Nursing students indicate they learn from their performances and enjoy the experience. The SCEs allows them to practice without a live patient in case they made a mistake, to learn from their mistakes, and learn to prioritize nursing care.

Negative comments on the evaluations were a desire to do more SCEs so they can perform different nursing roles. The nursing students want more preparation time with intravenous pumps and fetal monitoring. They want to be able to complete more SCEs prior to hospital clinical rotations.

Overall, the SCEs accomplish what they were intended to do. Nursing care performance improved during the hospital clinical rotations and grades on lecture testing improved.

\section{References}

1. http://www.aacnnursing.org/News-Information/Fact-Sheets/ Nursing-Shortage.

2. https://www.bls.gov/ooh/healthcare/registered-nurses.htm.

3. Alexander M. NCSBN simulation guidelines for prelicensure nursing programs. Journal of Nursing Regulation. 2015; 6: 39-42.

4. Spector N, Hooper JI, Silvestre JI, et al. Board of nursing approval of registered nurse education programs. Journal of Nursing Regulation. 2018; 8: 22-29. 


\section{Appendix A}

\section{Student Objectives for SCEs of Patient with Gestational Diabetes Mellitus (GDM)}

The student will identify pertinent assessment findings related to the patient with gestational diabetes (GDM) and prioritize the plan of care.

The student will demonstrate safe medication administration according to the six rights of medication administration.

The student will demonstrate the skill components of inserting and intravenous catheter and indwelling urinary catheterization.

The student will demonstrate Leopold's maneuver, initiation of fetal monitoring, and the appropriate nursing interventions required based on the correct interpretation of the fetal monitoring strips.

The student will analyze laboratory and diagnostic test results.

The student will provide support and teaching to the woman and her family while in labor.

The student will assist with the delivery and care of the neonate born to the mother with GDM.

The student will identify abnormal postpartum complications associated with patients with gestational diabetes and prioritize the plan of care.

The student will effectively communicate with the healthcare team utilizing the ISBAR tool.

\section{Guidelines for Student Preparation}

For student success, a review of the following are required prior to the SCEs:

Medications (not all inclusive): Humulin regular insulin, RhoGAM, butorphanol, methylergonovine maleate, misoprostol, carboprost tromethamine, oxytocin, oxycodone/acetaminophen, ibuprofen, acetaminophen, MMR II vaccine, medroxyprogesterone, benzoczine spray, pramoxine.

\section{Appendix B}

\section{Performance Evaluation for GDM L\&D SCEs}

\begin{tabular}{|c|c|c|c|c|}
\hline Event & Pass & Fail & N/A & Comments \\
\hline \multicolumn{5}{|l|}{ Washes hands } \\
\hline \multicolumn{5}{|l|}{ Identifies team and verifies patient using two identifiers } \\
\hline \multicolumn{5}{|l|}{ Performs Leopold's maneuver and places on monitor } \\
\hline \multicolumn{5}{|l|}{ Obtains vital signs } \\
\hline \multicolumn{5}{|l|}{ Performs complete physical assessment } \\
\hline \multicolumn{5}{|l|}{ Correctly assesses fetal monitor } \\
\hline \multicolumn{5}{|l|}{ Starts IV and obtains labs } \\
\hline \multicolumn{5}{|l|}{ Administers ordered sc insulin on admission } \\
\hline \multicolumn{5}{|l|}{$\begin{array}{c}\text { Assesses bladder status; indwelling cath for specimen after obtaining } \\
\text { order }\end{array}$} \\
\hline \multicolumn{5}{|l|}{ Obtains hourly fingerstick blood glucose } \\
\hline \multicolumn{5}{|l|}{$\begin{array}{l}\text { Performs med calc and starts insulin drip and adjusts insulin drip } \\
\text { accordingly }\end{array}$} \\
\hline \multicolumn{5}{|l|}{ Assesses pain and administers pain meds after obtaining orders } \\
\hline \multicolumn{5}{|l|}{ Assesses fetus for reactivity after pain med } \\
\hline \multicolumn{5}{|l|}{ Notifies provider of assessment and lab results } \\
\hline \multicolumn{5}{|l|}{ Prepares bed for delivery } \\
\hline \multicolumn{5}{|l|}{ Assists with pushing/delivery } \\
\hline Provides emotional support to patient throughout simulation & & & & \\
\hline
\end{tabular}




\section{Appendix C}

Performance Evaluation for GDM Postpartum SCEs

\begin{tabular}{|c|c|c|c|c|}
\hline Event & Pass & Fail & N/A & Comments \\
\hline \multicolumn{5}{|l|}{ Washes hands } \\
\hline \multicolumn{5}{|l|}{ Identifies team and verifies patient using two identifiers } \\
\hline \multicolumn{5}{|l|}{ Obtains vital signs } \\
\hline \multicolumn{5}{|l|}{ Performs complete physical assessment (BUBBLE-HE) } \\
\hline \multicolumn{5}{|l|}{ Starts IVs and correctly calculate rates } \\
\hline \multicolumn{5}{|l|}{ Obtains labs and calls provider } \\
\hline \multicolumn{5}{|l|}{ Performs fingerstick BS and adm sc insulin } \\
\hline \multicolumn{5}{|l|}{$\begin{array}{l}\text { Assesses bladder status and in \& out catheter to patient per } \\
\text { prn orders }\end{array}$} \\
\hline \multicolumn{5}{|l|}{ Assesses pain and medicates prn according to 6 rights } \\
\hline \multicolumn{5}{|l|}{ Administers RhoGAM IM } \\
\hline \multicolumn{5}{|l|}{ Immediate actions after hemorrhage: } \\
\hline \multicolumn{5}{|l|}{ Identifies changes in patient condition } \\
\hline \multicolumn{5}{|l|}{ Changes patient's bed position } \\
\hline \multicolumn{5}{|l|}{ Assesses fundus and bleeding } \\
\hline \multicolumn{5}{|l|}{ Assesses vital signs, LOC } \\
\hline \multicolumn{5}{|l|}{$\begin{array}{l}\text { Calls provider with assessment using ISBAR and obtains } \\
\text { orders }\end{array}$} \\
\hline \multicolumn{5}{|l|}{ Administers ordered medications using 6 rights } \\
\hline \multicolumn{5}{|l|}{ Increases IV fluids and administers $\mathrm{O} 2$} \\
\hline \multicolumn{5}{|l|}{ Continually assesses fundus/bleeding } \\
\hline \multicolumn{5}{|l|}{ Monitors vital signs, pulse oximetry, LOC } \\
\hline \multicolumn{5}{|l|}{ Provides emotional support throughout SCE } \\
\hline \multicolumn{5}{|l|}{ Identifies improvement in patient status } \\
\hline $\begin{array}{l}\text { Documents in nursing notes, MAR and any other pertinent } \\
\text { areas of medical record }\end{array}$ & & & & \\
\hline
\end{tabular}

\section{Appendix D}

Simulated Clinical Experience Evaluation Results

\begin{tabular}{|c|c|c|c|c|c|}
\hline Date: & Various Dates & Course: & NUR 220 & Semester: & 2018 \\
\hline & Instructors: & \multicolumn{4}{|c|}{ Cheryl DeGraw, Jessica Jones, Ashley Branham } \\
\hline
\end{tabular}

\begin{tabular}{|c|c|c|c|c|}
\hline Participation in the simulated clinical experience: & Strongly Agree & Agree & Undecided & Disagree \\
\hline Improved my ability to function effectively as a team member through collaboration. & $83.33 \%$ & $16.67 \%$ & & \\
\hline Encouraged me to use my critical thinking/decision making skills. & $77.78 \%$ & $22.22 \%$ & & \\
\hline $\begin{array}{l}\text { Improved my ability to communicate with patients/other members of the health care } \\
\text { team. }\end{array}$ & $75.00 \%$ & $25.00 \%$ & & \\
\hline Improved my ability to identify and prioritize patient safety needs. & $83.33 \%$ & $16.67 \%$ & & \\
\hline Improved my assessment and evaluation skills by using the nursing process. & $75.00 \%$ & $25.00 \%$ & & \\
\hline
\end{tabular}




\begin{tabular}{|c|c|c|c|c|}
\hline Prompted me to prioritize and implement evidence-based nursing interventions. & $83.33 \%$ & $16.67 \%$ & & \\
\hline $\begin{array}{l}\text { Improved my understanding of medication administration \& the nursing implications } \\
\text { that apply to each administered medication. }\end{array}$ & $77.14 \%$ & $22.86 \%$ & & \\
\hline Guided my learning of the theory content to be applied in the clinical setting. & $80.56 \%$ & $19.44 \%$ & & \\
\hline Improved my confidence to function competently in the clinical setting. & $75.00 \%$ & $22.22 \%$ & $2.78 \%$ & \\
\hline Allowed me to effectively delegate to my team members. & $72.22 \%$ & $22.22 \%$ & $5.56 \%$ & \\
\hline $\begin{array}{l}\text { Improved my communication skills with members of the health care team by using } \\
\text { the ISBAR format. }\end{array}$ & $77.78 \%$ & $22.22 \%$ & & \\
\hline Improved my professional behaviors and leadership skills. & $75.00 \%$ & $22.22 \%$ & $2.78 \%$ & \\
\hline The objectives of the simulation clearly stated. & $75.00 \%$ & $25.00 \%$ & & \\
\hline Simulation was appropriate for my level of learning. & $75.00 \%$ & $25.00 \%$ & & \\
\hline $\begin{array}{l}\text { I can apply what I learned in this simulation to enhance my understanding of the } \\
\text { content presented in lecture. }\end{array}$ & $77.78 \%$ & $22.22 \%$ & & \\
\hline $\begin{array}{l}\text { Do you think the simulated clinical experience was appropriately scheduled in the } \\
\text { course? }\end{array}$ & YES & $84.38 \%$ & NO & $15.63 \%$ \\
\hline
\end{tabular}

PLEASE PROVIDE SUGGESTIONS FOR IMPROVING THE SIMULATED CLINICAL EXPERIENCE?

WHAT WOULD YOU LIKE TO DO DIFFERENTLY IF GIVEN THE OPPORTUNITY?

\section{ADDITIONAL COMMENTS}

() 2019 Cheryl L. DeGraw. This article is distributed under the terms of the Creative Commons Attribution 4.0 International License 